\title{
Mapeamento do fósforo adsorvido por meio da cor e da suscetibilidade magnética do solo
}

\author{
Rafael Gonçalves Peluco(1), José Marques Júnior(1), Diego Silva Siqueira(1), Gener Tadeu Pereira(1), \\ Ronny Sobreira Barbosa(2) e Daniel de Bortoli Teixeira ${ }^{(1)}$
}

\begin{abstract}
(1)Universidade Estadual Paulista, Departamento de Solos e Adubos, Via de Acesso Prof. Paulo Donato Castellane, s/no, CEP 14884-900 Jaboticabal, SP, Brasil. E-mail: rgpeluco@agronomo.eng.br, marques@fcav.unesp.br, diego_silvasiqueira@yahoo.com.br, genertp@fcav.unesp.br, daniel.dbt@hotmail.com (2)Universidade Federal do Piauí, Campus Professora Cinobelina Elvas, Rodovia Municipal Bom Jesus-Viana, Km 01, Planalto Horizonte, s/no, CEP 64900-000 Bom Jesus, PI, Brasil. E-mail: ronny.barbosa@ufpi.edu.br
\end{abstract}

Resumo - O objetivo deste trabalho foi avaliar a possibilidade de se mapear o fósforo adsorvido por meio da avaliação da cor e da suscetibilidade magnética do solo. O experimento foi realizado em área de cana-de-açúcar com 380 ha, que compreendia quatro tipos de Latossolos, com diferentes texturas e fertilidades naturais. Foram coletadas 241 amostras de solo, na profundidade de 0,00-0,25 m. O índice de avermelhamento do solo foi calculado a partir dos valores de matiz, valor e croma das amostras, obtidos com espectroscopia de reflectância difusa. Foram ajustados modelos de regressão entre o fósforo adsorvido e a suscetibilidade magnética ou o índice de avermelhamento dos solos. A dependência espacial dos dados foi avaliada por meio de geoestatística. A suscetibilidade magnética correlaciona-se significativamente ao fósforo adsorvido, o que possibilita seu uso como componente de funções de pedotransferência para quantificação indireta do fósforo adsorvido ao solo. $\mathrm{O}$ índice de avermelhamento e a suscetibilidade magnética apresentam dependência espacial com o fósforo adsorvido. A suscetibilidade magnética e o índice de avermelhamento dos solos, avaliado pela espectroscopia de reflectância difusa, podem auxiliar no mapeamento e na identificação de áreas com diferentes potenciais de adsorção de fósforo.

Termos para indexação: avaliação da fertilidade do solo, dependência espacial, espectroscopia de reflectância difusa, geoestatística, pedotransferência, variabilidade espacial.

\section{Mapping adsorbed phosphorus through soil color and magnetic susceptibility}

Abstract - The objective of this work was to evaluate the possibility of mapping adsorbed phosphorus through soil color and magnetic susceptibility. The experiment was done in a 380-ha sugarcane area, which included four types of Oxisols, with different texture and natural fertility. Two hundred and forty-one soil samples were collected at $0.00-0.25-\mathrm{m}$ depth. The soil redness index was calculated from sample values of hue, value, and chroma, obtained with diffuse reflectance spectroscopy. Regression models were adjusted between adsorbed phosphorus and soil magnetic susceptibility or redness index. Data spatial dependence was evaluated with geostatistics. Magnetic susceptibility is significantly correlated to adsorbed phosphorus, which allows it to be used as a component in pedotransfer functions for indirect quantification of soil adsorbed phosphorus. The redness index and the magnetic susceptibility show spatial dependency with adsorbed phosphorus. Soil magnetic susceptibility and redness index, evaluated with diffuse reflectance spectroscopy, can aid in the mapping and identification of areas with different phosphorus adsorption potential.

Index terms: soil fertility evaluation, spatial dependency, diffuse reflectance spectroscopy, geostatistics, pedotransfer, spatial variability.

\section{Introdução}

A predição e o mapeamento da variabilidade espacial de atributos do solo possibilitam racionalizar o manejo agrícola e concorrem para o aumento da sustentabilidade da agricultura. No entanto, estudos com esse objetivo requerem a retirada e a análise de grande número de amostras e, portanto, têm alto custo (Demattê et al., 2007).
A utilização de funções de pedotransferência, que estimem indiretamente atributos do solo, é uma alternativa interessante para redução de custos e do tempo gasto nesses estudos. Segundo McBratney et al. (2002), funções de pedotransferência podem ser definidas como modelos matemáticos utilizados para estimar atributos do solo a partir de outros atributos facilmente medidos, como a suscetibilidade magnética (SM) (Siqueira et al., 2010) e a cor medida por 
espectroscopia de reflectância difusa (ERD) (Torrent \& Barrón, 2008).

A SM pode ser definida como a medida da facilidade com que um material se magnetiza na presença de campo magnético. Essa propriedade depende das concentrações e das características (estrutura magnética do cristal, composição, tamanho, morfologia e domínio) dos minerais presentes na rocha ou no solo (Verosub \& Robert, 1995), e pode ser considerada como um micromarcador de atributos do solo. Desse modo, é possível estabelecer correlações entre a SM e os atributos do solo (Mathé et al., 2006).

A cor do solo é resultante da capacidade dos pigmentos em absorver mais ou menos a luz de diferentes comprimentos de onda (Barrón et al., 2000). De acordo com Torrent \& Barrón (2008), o uso da ERD consiste na aquisição quali-quantitativa de dados por meio da transmissão ou da absorção da energia radiante incidente em uma amostra. A energia diferencial dos vários comprimentos de onda do espectro é absorvida pelas moléculas em estudo, e a análise quali-quantitativa da amostra é realizada com base na Lei de Beer-Lambert.

A SM (Siqueira et al., 2010) e a ERD (Torrent \& Barrón, 2008), bem como o uso dessas técnicas em conjunto (Marques Junior et al., 2014), podem auxiliar no estudo de atributos mineralógicos, físicos e químicos do solo.

O teor de $\mathrm{P}$ disponível no solo tem sido relatado como a limitação nutricional mais generalizada à produção agrícola (Johnson \& Loeppert, 2006), principalmente em solos intemperizados. Nesses solos, a eficiência da adubação fosfatada é baixa, pois grande parte do $\mathrm{P}$ adicionado é imobilizada no solo, em virtude de reações de precipitação, adsorção e fixação em coloides minerais. Além disso, o P também é facilmente convertido para a fração orgânica do solo (Busato et al., 2005). Rolim Neto et al. (2004) ressaltam que o suprimento natural de $P$ é insatisfatório ao adequado crescimento das plantas, na maioria dos solos brasileiros.

Assim, o conhecimento da variabilidade espacial do potencial de adsorção de $\mathrm{P}$ nos solos pode auxiliar no planejamento agrícola, uma vez que o conhecimento da variabilidade espacial de atributos do solo permite a recomendação mais eficiente e econômica de fertilizantes e corretivos (Barbieri et al., 2008).

O objetivo deste trabalho foi avaliar a possibilidade de se mapear o fósforo adsorvido por meio da avaliação da cor e da suscetibilidade magnética do solo.

\section{Material e Métodos}

$\mathrm{O}$ estudo foi realizado em área com lavoura comercial de cana-de-açúcar, de 380 ha, na Usina São Martinho, no nordeste do Estado de São Paulo, no Município de Guatapará $\left(21^{\circ} 29^{\prime} 48^{\prime \prime S}, 48^{\circ} 02^{\prime} 16^{\prime \prime} \mathrm{W}\right.$, a $557 \mathrm{~m}$ de altitude média). A região apresenta clima do tipo Aw megatérmico, tropical com estação seca de inverno, segundo Köppen, e precipitação média de $1.400 \mathrm{~mm}$, com chuvas concentradas no período de novembro a fevereiro. A área recebe adubação fosfatada há mais de uma década e está inserida na província geomorfológica do Planalto Ocidental Paulista, próximo ao limite das Cuestas Basálticas, no divisor litoestratigráfico arenito-basáltico.

Os solos, na área de estudo, foram classificados como Latossolo Vermelho distrófico, de textura média (LVd3.1; LVd3.4); Latossolo Vermelho distroférrico, de textura argilosa (LVdf1.1); Latossolo Vermelho eutroférrico, de textura argilosa (LVef1.1); e Latossolo Vermelho-Amarelo distrófico, de textura média (LVAd3.1) (Santos et al., 2006). Foram coletadas 86 amostras de solo a cada $30 \mathrm{~m}$, em uma transeção de $2.600 \mathrm{~m}$, da maior para a menor altitude da área, no caminho de menor declividade, o que permitiu obter amostras de todas as classes de solo avaliadas. Outros 155 pontos também foram amostrados em uma malha regular com densidade de 1 ponto a cada 2,5 ha. Dessa forma, o total de amostras obtido foi de 241, sempre na profundidade de $0,00-0,25 \mathrm{~m}$. Todas as amostras foram secas e passadas em peneira de $2,0 \mathrm{~mm}$ de abertura de malha, para análises granulométricas e químicas, e para as medições de ERD e SM.

A granulometria do solo foi determinada pela metodologia proposta por Claessen (1997), e a SM das amostras foi obtida com medidor MS2 (Bartington Instruments Ltd., Oxford, Inglaterra), acoplado a um sensor MS2B de duas frequências, conforme descrito por Dearing (1999).

Para representar as classes de solo da área experimental, foram escolhidos, de maneira aleatória, 12 pontos da transeção, nos quais foram obtidos os teores de fósforo adsorvido (Alvarez V. et al., 2000), utilizados na análise de regressão com SM e ERD.

Para a obtenção dos espectros de reflectância difusa, foi utilizado o sensor de laboratório Lambda 950 (Perkin Elmer Ltd., Waltham, MA, EUA). Aproximadamente $0,5 \mathrm{~g}$ de terra fina seca ao ar (TFSA) foi moído em ágata, até coloração constante. 
O conteúdo foi colocado em porta-amostras com um espaço cilíndrico de $16 \mathrm{~mm}$. Os valores de reflectância foram determinados em espectrofotômetro equipado com esfera integradora de $80 \mathrm{~mm}$, a cada $1 \mathrm{~nm}$, com tempo de integração de $0,2 \mathrm{~s}$ e varredura no intervalo de 380 a $780 \mathrm{~nm}$. Após a obtenção dos espectros de reflectância difusa das amostras, foram determinados os valores de triestímulo XYZ definidos pela Comisión Internacional de L'Eclairage (Wyszecki \& Stiles, 1982). A partir das coordenadas XYZ, foram deduzidos os valores Munsell de matiz, valor e croma, com uso do programa Munsell Conversion, versão 6.4 (Barrón et al., 2000). Com base nos valores de matiz, valor e croma, calculou-se o índice de avermelhamento (IAV), de acordo com a equação (Barrón et al., 2000): $\mathrm{IAV}=[(10-\mathrm{Matiz}) \times \mathrm{Croma}] /$ Valor.

Os dados foram submetidos à análise estatística descritiva, tendo-se determinado média, mediana, coeficiente de variação, valores máximo e mínimo, além da assimetria e da curtose. Os modelos de regressão entre a $\mathrm{SM}$, o IAV e o $\mathrm{P}$ adsorvido foram obtidos com o programa estatístico Minitab, versão 13.1 (Minitab, State College, PA, EUA). Quando houve correlação entre os valores de SM e ERD com os teores de $\mathrm{P}$ adsorvido ao solo, eles foram utilizados em função de pedotransferência para estimar, por meio do modelo de regressão, os teores de $\mathrm{P}$ adsorvido nos demais pontos amostrais.

Com base na carta de solos da área experimental (escala detalhada de 1:12.000), elaborada pelo Centro de Tecnologia Canavieira, foram calculadas as médias dos atributos estudados, a partir dos valores obtidos nas amostras retiradas em cada classe de solo. Os teores médios foram comparados pelo teste de Tukey, a 5\% de probabilidade.

A análise da dependência espacial dos dados foi feita por meio da geoestatística (Vieira et al., 1983), tendose utilizado variogramas modelados com o auxílio do programa GS+: geostatistics for environmental sciences (Robertson, 1998), e os modelos matemáticos foram ajustados aos variogramas experimentais, a partir dos quais foi determinado o grau de dependência espacial de cada atributo estudado. Os valores interpolados por meio da krigagem foram usados para construção de mapas dos atributos avaliados, com uso do programa Surfer, versão 7.0 (Golden Software, New York, EUA). As correlações espaciais entre os atributos estudados foram analisadas por variogramas cruzados.
Os variogramas experimentais foram escolhidos com base no número de pares envolvidos no cálculo da semivariância dos primeiros "lags", na presença de patamar claramente definido e no valor de $\mathrm{R}^{2}$, para $\mathrm{o}$ modelo ajustado e para a validação cruzada.

\section{Resultados e Discussão}

Observou-se correlação positiva da SM com o P adsorvido (Figura 1), o que possibilita sua utilização como componente de funções de pedotransferência, para a quantificação indireta dos teores de $\mathrm{P}$ adsorvido ao solo. Siqueira et al. (2010) também encontraram correlação positiva e significativa entre esses atributos em Argissolo. Verosub \& Roberts (1995) concluíram que a SM tem relação direta com a mineralogia do solo e pode ser afetada pela presença de óxidos de ferro secundários, que são os principais responsáveis pela adsorção de P (Camargo et al., 2012).
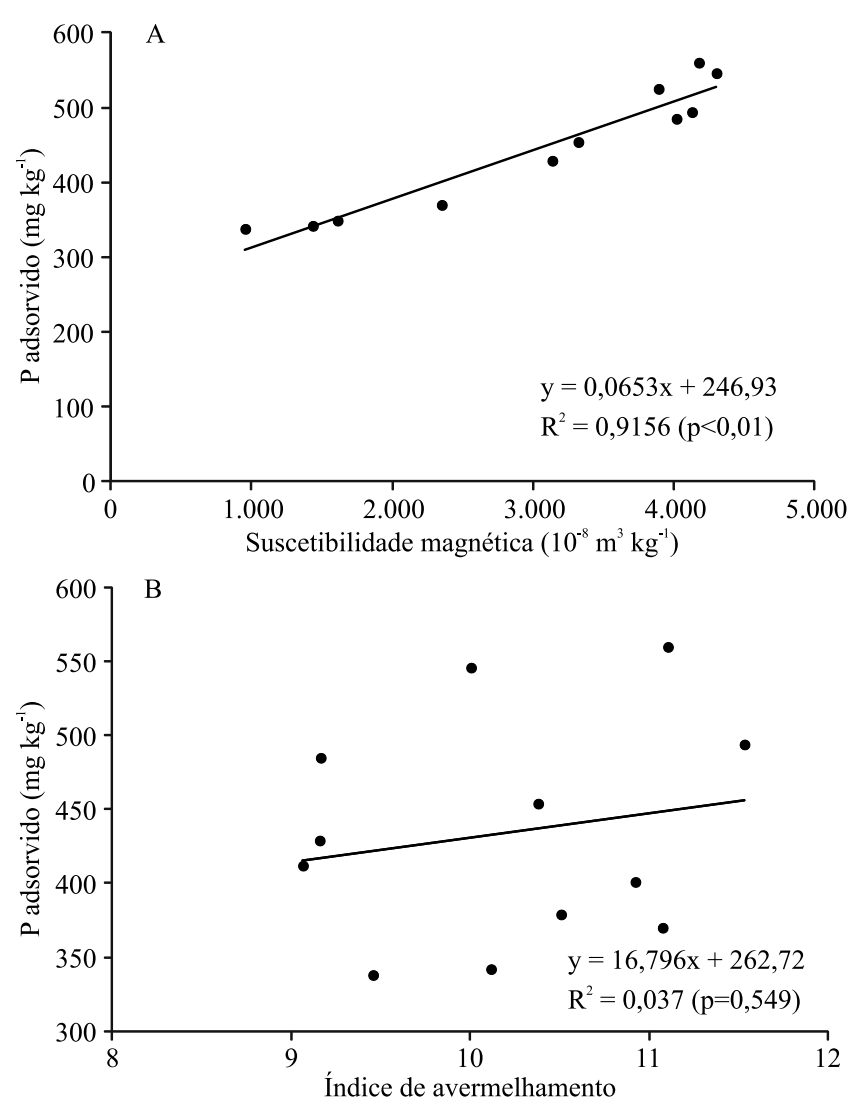

Figura 1. Modelos de regressão entre o $\mathrm{P}$ adsorvido e a suscetibilidade magnética (SM) ou o índice de avermelhamento (IAV) dos solos, nos 12 pontos da transeção. 
O IAV, no entanto, não se correlacionou significativamente ao $\mathrm{P}$ adsorvido (Figura 1), o que inviabiliza, neste estudo, seu uso em funções de pedotransferência para estimativa do P adsorvido. Almeida et al. (2003), no entanto ao avaliar os atributos mineralógicos de Latossolos por meio da cor dos solos, constataram correlações significativas com o P adsorvido ao solo.

De acordo com a classificação de Warrick \& Nielsen (1980), os valores de IAV apresentaram baixa variabilidade $(\mathrm{CV}<12 \%)$; os de $\mathrm{P}$ adsorvido tiveram variabilidade moderada $(12 \%<\mathrm{CV}<24 \%)$; e os teores de argila e a $\mathrm{SM}$ apresentaram alta variabilidade $(\mathrm{CV}>24 \%)$, com os maiores valores de CV obtidos para SM (Tabela 1). Essa alta variabilidade da SM reflete a grande sensibilidade deste atributo para indicar processos pedogenéticos. Mathé et al. (2006) afirmam que a SM pode ser utilizada como microindicadora das condições do solo.

O IAV apresentou o menor CV (Tabela 1), o que possivelmente esteve relacionado à predominância de Latossolos Vermelhos na área experimental. Novaes Filho et al. (2007) relatam que há correspondência satisfatória entre os intervalos de IAV nos horizontes diagnósticos e a nomenclatura do segundo nível categórico (subordem) de Argissolos e Latossolos, conforme o Sistema Brasileiro de Classificação dos Solos (Santos et al., 2006). Portanto, esses intervalos, apesar de diferentes, são semelhantes em solos vermelho-amarelos e vermelhos.

As maiores amplitudes observadas nos valores de SM e no teor de argila (Tabela 1) indicam variação considerável nos materiais de origem dos solos (Fontes et al., 2000), na área do experimento. Essa indicação está de acordo com o fato de a área experimental estar inserida em um divisor litoestratigráfico arenito-basáltico.

As médias e as medianas dos atributos estudados foram próximas, e os valores de assimetria e curtose foram próximos à zero (Tabela 1). Dessa forma, os dados apresentaram distribuição simétrica e mostraramse adequados para uso em técnicas geoestatísticas (Warrick \& Nielsen, 1980).
Os solos LVef1.1 e LVdf1.1, mais argilosos, apresentaram maiores valores de IAV do que os LVd3.4 e LVAd3.1, de textura média (Tabela 2). Novaes Filho et al. (2007) encontraram diferenças entre os valores de intervalos de classes do IAV de Latossolos Vermelhos, Vermelho-Amarelos e Amarelos, em microbacias na Amazônia regional.

Os valores de $\mathrm{SM}$ e de $\mathrm{P}$ adsorvido foram maiores nos Latossolos Vermelhos férricos argilosos (Tabela 2). Segundo Camargo et al. (2012), a adsorção de fósforo nos solos está fortemente relacionada aos conteúdos de óxidos de ferro e de argila, que também influenciam diretamente o comportamento magnético dos solos (Fontes et al., 2000).

Os atributos avaliados apresentaram dependência espacial, expressa nos ajustes dos modelos aos variogramas (Vieira et al., 1983) (Tabela 3). O grau de dependência espacial (GDE) dos variogramas foi inferior a $25 \%$, isto é, mostrou dependência espacial forte, conforme Cambardella et al. (1994). De acordo com esses autores, valores de GDE menores ou iguais a $25 \%$ indicam dependência espacial forte; entre 25 e $75 \%$, média; e maior que $75 \%$, fraca.

Os modelos ajustados aos variogramas foram o exponencial, para o $\mathrm{P}$ adsorvido e a argila, e o esférico, para a SM e o IAV. Silva et al. (2013) e Marques Junior et al. (2014) também verificaram ajustes dos variogramas de SM e IAV ao modelo esférico. Segundo Burgess \& Webster (1980), esse modelo é associado à representação da continuidade espacial de atributos com características de transição abrupta, que são mais facilmente identificados no campo, enquanto atributos representados pelo modelo exponencial ou gaussiano apresentam transição mais sutil.

O alcance é um importante parâmetro dos variogramas, e representa a distância máxima em que uma variável está correlacionada espacialmente à outra; ou seja, avaliações com distâncias maiores que o alcance têm distribuição aleatória e, portanto, são independentes. Neste caso, deve-se aplicar a estatística

Tabela 1. Estatística descritiva dos atributos estudados nos 241 pontos de $\operatorname{coleta}^{(1)}$.

\begin{tabular}{|c|c|c|c|c|c|c|c|}
\hline Atributo & Média & Mediana & $\mathrm{CV}(\%)$ & Mínimo & Máximo & Assimetria & Curtose \\
\hline Argila $\left(\mathrm{g} \mathrm{kg}^{-1}\right)$ & 425,41 & 454,50 & 28,88 & 83,00 & 619,50 & $-0,61$ & $-0,48$ \\
\hline $\mathrm{P}$ adsorvido $\left(\mathrm{g} \mathrm{kg}^{-1}\right)$ & 443,81 & 455,89 & 23,98 & 257,90 & 733,94 & 0,03 & $-0,82$ \\
\hline Índice de avermelhamento (IAV) & 10,29 & 10,25 & 11,15 & 6,20 & 13,32 & $-0,10$ & 0,35 \\
\hline $\mathrm{SM}\left(10^{-8} \mathrm{~m}^{3} \mathrm{~kg}^{-1}\right)$ & $3.015,00$ & $3.200,00$ & 54,06 & 168,00 & $7.458,00$ & 0,03 & $-0,82$ \\
\hline
\end{tabular}

${ }^{(1)} \mathrm{SM}$, suscetibilidade magnética; e CV, coeficiente de variação. 
clássica. $\mathrm{O} \mathrm{P}$ adsorvido foi $\mathrm{o}$ atributo com maior alcance, e o IAV, com o menor (Tabela 3).

Para averiguar a distribuição e a correlação espacial entre os atributos, foram construídos

Tabela 2. Médias dos atributos estudados em cada classe de solo $^{(1)}$.

\begin{tabular}{lccccc}
\hline $\begin{array}{l}\text { Classe de } \\
\text { solo }\end{array}$ & $\begin{array}{c}\text { Área } \\
\text { (ha) }\end{array}$ & $\begin{array}{c}\mathrm{SM}\left(10^{-8}\right. \\
\left.\mathrm{m}^{3} \mathrm{~kg}^{-1}\right)\end{array}$ & IAV & $\begin{array}{c}\text { Argila } \\
\left(\mathrm{g} \mathrm{kg}^{-1}\right)\end{array}$ & $\begin{array}{c}\text { P adsorvido } \\
\left(\mathrm{mg} \mathrm{kg}^{-3}\right)\end{array}$ \\
\hline LVAd3.1 & 7 & $952 \mathrm{~cd}$ & $9,35 \mathrm{~b}$ & $315 \mathrm{~b}$ & $309 \mathrm{~cd}$ \\
LVd3.1 & 126 & $1.728 \mathrm{c}$ & $10,24 \mathrm{ab}$ & $361 \mathrm{~b}$ & $359 \mathrm{c}$ \\
LVd3.4 & 47 & $633 \mathrm{~d}$ & $9,58 \mathrm{~b}$ & $207 \mathrm{c}$ & $288 \mathrm{~d}$ \\
LVdf1.1 & 107 & $3.841 \mathrm{~b}$ & $10,45 \mathrm{a}$ & $477 \mathrm{a}$ & $497 \mathrm{~b}$ \\
LVef1.1 & 93 & $4.510 \mathrm{a}$ & $10,48 \mathrm{a}$ & $512 \mathrm{a}$ & $541 \mathrm{a}$ \\
\hline
\end{tabular}

(1)Médias seguidas de letras iguais não diferem pelo teste de Tukey, a 5\% de probabilidade. LVd, Latossolo Vermelho distrófico; LVef, Latossolo Vermelho eutroférrico; LVAd, Latossolo Vermelho-Amarelo distrófico; LVdf, Latossolo Vermelho distroférrico; SM, suscetibilidade magnética; e IAV, índice de avermelhamento.
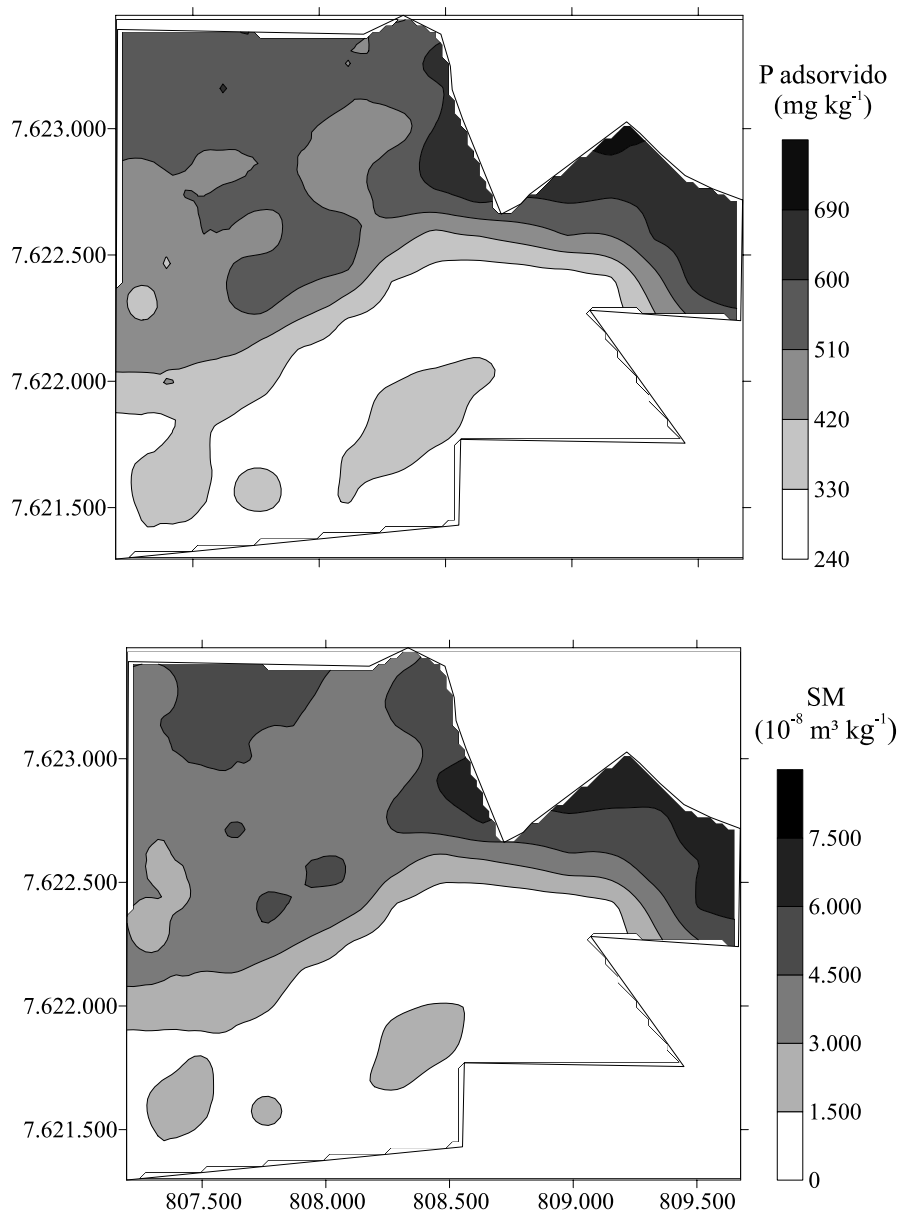

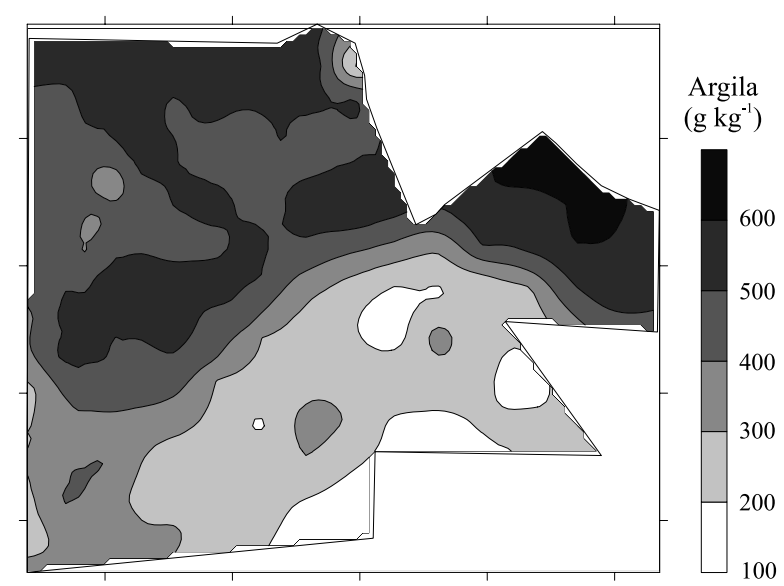

mapas de krigagem (Figura 2) e variogramas cruzados (Tabela 3). Houve grande similaridade na variabilidade espacial entre os mapas de teor de argila, $\mathrm{SM}$ e $\mathrm{P}$ adsorvido. Esse resultado foi validado pelos variogramas cruzados, que apresentaram correlação espacial definida positiva entre esses atributos (Figura

Tabela 3. Geoestatística para os teores de P adsorvido e de argila, e para os valores de suscetibilidade magnética (SM) e índice de avermelhamento (IAV) dos solos.

\begin{tabular}{lcccccc}
\hline Variável & Modelo & $\begin{array}{c}\text { Efeito } \\
\text { pepita }\left(\mathrm{C}_{0}\right)\end{array}$ & $\begin{array}{c}\text { Patamar } \\
\left(\mathrm{C}_{0}+\mathrm{C}_{1}\right)\end{array}$ & $\begin{array}{c}\text { Alcance } \\
(\mathrm{m})\end{array}$ & $\begin{array}{c}\text { GDE } \\
(\%)^{(1)}\end{array}$ & $\mathrm{R}^{2}$ \\
\hline $\mathrm{P}_{\text {adsorvido }}$ & Exponencial & 20,00 & 4.958 & 1.395 & 0,40 & 0,99 \\
Argila & Exponencial & 7,40 & 97,40 & 1.284 & 7,60 & 0,98 \\
$\mathrm{IAV}$ & Esférico & 0,20 & 1,39 & 464 & 14,57 & 0,89 \\
$\mathrm{SM}$ & Esférico & 140.000 & 1.171 .000 & 1.136 & 11,95 & 0,96 \\
\hline
\end{tabular}

${ }^{(1)} \mathrm{GDE}$, grau de dependência espacial $\left[\mathrm{C}_{0} /\left(\mathrm{C}_{0}+\mathrm{C}_{1}\right)\right] \times 100$.

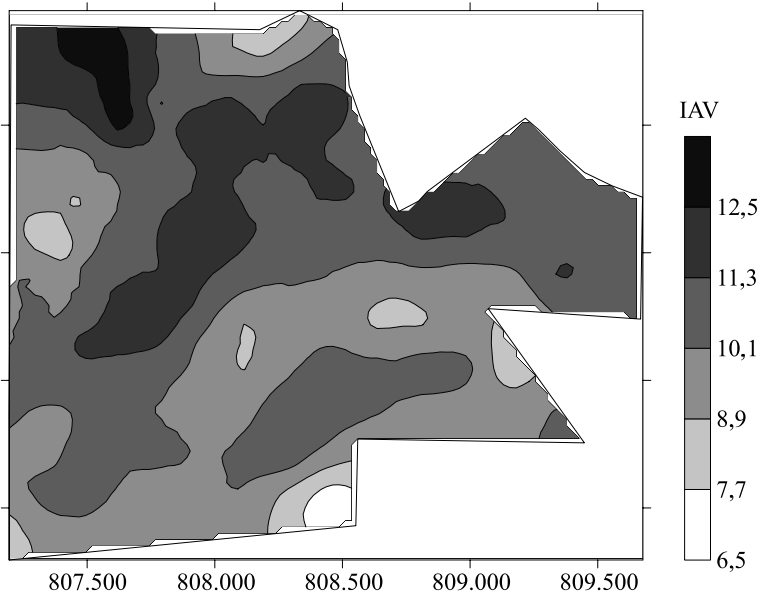

Figura 2. Mapas de krigagem de $\mathrm{P}$ adsorvido, argila, sucetibilidade magnética (SM) e índice de avermelhamento (IAV). 
3), com forte grau de dependência espacial entre o P adsorvido e o teor de argila, e entre a argila e a SM; e médio grau de dependência entre o $\mathrm{P}$ adsorvido e a SM.
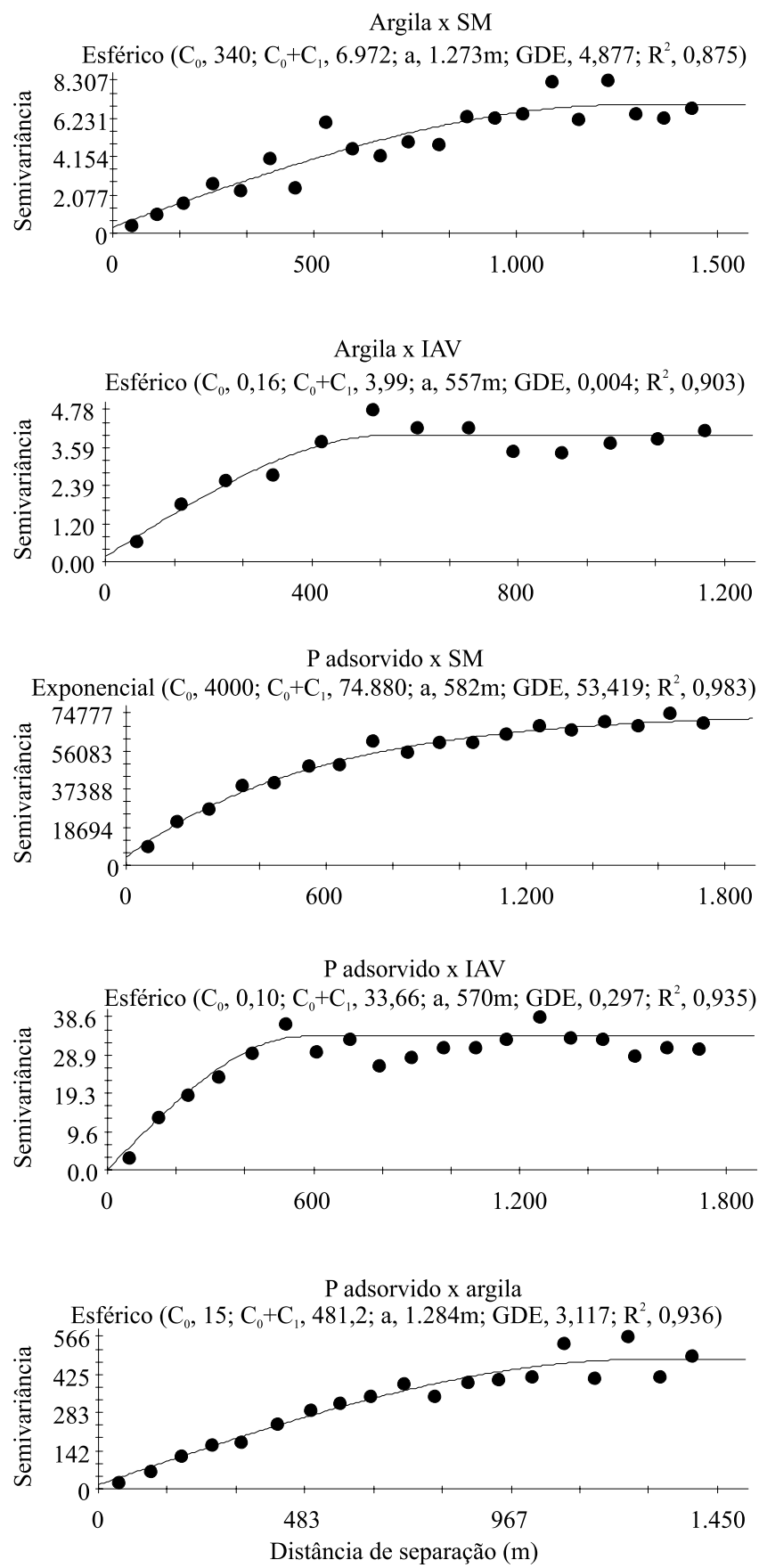

Figura 3. Variogramas cruzados entre os teores de $\mathrm{P}$ adsorvido e os de argila, em função da suscetibilidade magnética (SM) e do índice de avermelhamento (IAV) dos solos.
Os solos mais argilosos apresentaram zonas de maior adsorção de P. Barbieri et al. (2013) também obtiveram correlação espacial positiva entre o teor de argila e o P adsorvido, em Latossolo Vermelho eutroférrico, sob cultivo de cana-de-açúcar. Valladares et al. (2003) apontam que a correlação entre esses atributos está fortemente relacionada à predominância de óxidos de ferro e alumínio, além de caulinita, na fração argila desses solos. Em condições de reação ácida a moderadamente ácida, os óxidos apresentam carga líquida positiva, com alta afinidade pela adsorção de ânions, especialmente o do fosfato.

Os maiores valores de SM também foram observados nos solos com maiores teores de argila (Figura 2). Fontes et al. (2000) ressaltam que a SM é maior em áreas mais argilosas, em razão da maior proporção de minerais magnéticos nessa classe textural. Uma vez que os principais minerais responsáveis pela adsorção de $\mathrm{P}$ também são encontrados na fração argila, a correlação significativa entre SM e P adsorvido já era esperada.

$\mathrm{Na}$ análise dos mapas, foi possível verificar que as isolinhas no mapa de IAV são semelhantes às dos mapas de argila e de $\mathrm{P}$ adsorvido (Figura 2). Os maiores valores de IAV e de $\mathrm{P}$ adsorvido ocorreram nas áreas com maior teor de argila. De acordo com Almeida et al. (2003), o emprego da refletância difusa para a determinação dos espectros de cor é útil não só para a caracterização mais precisa da cor dos solos, mas, também, para a quantificação dos teores de óxidos de ferro na fração argila (Barrón et al., 2000). Desse modo, o IAV pode auxiliar no mapeamento e na identificação de áreas com maior potencial de adsorção de $\mathrm{P}$.

\section{Conclusões}

1. A suscetibilidade magnética correlaciona-se significativamente aos teores de fósforo adsorvido e pode ser utilizada como componente de função de pedotransferência, para quantificação indireta da adsorção desse elemento nos solos.

2. O índice de avermelhamento e a suscetibilidade magnética apresentam dependência espacial com o fósforo adsorvido.

3. A suscetibilidade magnética e a cor do solo, estimada pela espectroscopia de reflectância difusa, podem auxiliar no mapeamento e na identificação de áreas com diferentes potenciais de adsorção de fósforo. 


\section{Agradecimentos}

À Coordenação de Aperfeiçoamento de Pessoal de Nível Superior (Capes), pelo auxílio financeiro; e à Usina São Martinho S/A, pela concessão da área experimental e apoio na realização do experimento.

\section{Referências}

ALMEIDA, J.A.; TORRENT, J.; BARRÓN, V. Cor de solo, formas do fósforo e adsorção de fosfatos em Latossolos desenvolvidos de basalto do extremo-sul do Brasil. Revista Brasileira de Ciência do Solo, v.27, p.985-1002, 2003. DOI: 10.1590/ S0100-06832003000600003.

ALVAREZ V., V.H.; NOVAIS, F.R. de; DIAS, L.E.; OLIVEIRA, A.J. de. Determinação e uso do fósforo remanescente. Boletim Informativo da Sociedade Brasileira de Ciência do Solo, v.25, p.27-32, 2000.

BARBIERI, D.M.; MARQUES JUNIOR, J.; PEREIRA, G.T. Variabilidade espacial de atributos químicos de um Argissolo para aplicação de insumos à taxa variável em diferentes formas de relevo. Engenharia Agrícola, v.28, p.645-653, 2008. DOI: 10.1590/S0100-69162008000400004.

BARBIERI, D.M.; MARQUES JUNIOR, J.; PEREIRA, G.T.; LA SCALA JUNIOR, N.; SIQUEIRA, D.S.; PANOSSO, A.R. Comportamento dos óxidos de ferro da fração argila e do fósforo adsorvido, em diferentes sistemas de colheita de cana-de-açúcar. Revista Brasileira de Ciência do Solo, v.37, p.1557-1568, 2013. DOI: 10.1590/S0100-06832013000600012.

BARRÓN, V.; MELLO, J.W.V.; TORRENT, J. Caracterização de óxidos de ferro em solos por espectroscopia de reflectância difusa. In: NOVAIS, R.F. de; ALVAREZ V., V.H.; SCHAEFER, C.E.G.R. (Ed.). Tópicos em ciência do solo. Viçosa: Sociedade Brasileira de Ciência do Solo, 2000. v.1, p.139-162.

BURGESS, T.M.; WEBSTER, R. Optimal interpolation and isarithmic mapping of soil properties. I. The semi-variogram and punctual kriging. European Journal of Soil Science, v.31, p.315-331, 1980. DOI: 10.1111/j.1365-2389.1980.tb02084.x.

BUSATO, J.G.; CANELLAS, L.P.; VELlOSO, A.C.X. Fósforo num Cambissolo cultivado com cana-de-açúcar por longo tempo. I: Fracionamento seqüencial. Revista Brasileira de Ciência do Solo, v.29, p.935-945, 2005. DOI: 10.1590/S0100-06832005000600011.

CAMARGO, L.A.; MARQUES JÚNIOR, J.; PEREIRA, G.T.; ALLEONI, L.R.F. Spatial correlation between the composition of the clay fraction and contents of available phosphorus of an Oxisol at hillslope scale. Catena, v.100, p.100-106, 2012. DOI: 10.1016/j. catena.2012.07.016.

CAMBARDELLA, C.A.; MOORMAN, T.B.; NOVAK, J.M.; PARKIN, T.B.; KARLEN, D.L.; TURCO, R.F.; KONOPKA, A.E. Field-scale variability of soil properties in Central Iowa soils. Soil Science Society of America Journal, v.58, p.1501-1511, 1994. DOI: 10.2136/sssaj1994.03615995005800050033x.

CLAESSEN, M.E.C. (Org.). Manual de métodos de análise de solo. 2.ed. rev. atual. Rio de Janeiro: Embrapa-CNPS, 1997. 212p. (Embrapa-CNPS. Documentos, 1).
DEARING, J.A. Environmental magnetic susceptibility: using the Bartington MS2 system. $2^{\text {nd }}$ ed. Kenilworth: Chi Publishing, 1999. 54p.

DEMATTÊ, J.A.M.; GALDOS, M.V.; GUIMARÃES, R.; GENÚ, A.M.; NANNI, M.R.; ZULLO JUNIOR, J. Quantification of tropical soil attributes from ETM+/Landsat-7 data. International Journal of Remote Sensing, v.28, p.3813-3829, 2007. DOI: 10.1080/01431160601121469.

FONTES, M.P.F.; OLIVEIRA, T.S. de; COSTA, L.M. da; CAMPOS, A.A.G. Magnetic separation and evaluation of magnetization of Brazilian soils from different parent materials. Geoderma, v.96, p.81-99, 2000. DOI: 10.1016/S0016-7061(00)00005-7.

JOHNSON, S.E.; LOEPPERT, R.H. Role of organic acids in phosphate mobilization from iron oxide. Soil Science Society of America Journal, v.70, p.222-234, 2006. DOI: 10.2136/ sssaj2005.0012.

MARQUES JUNIOR, J.; SIQUEIRA, D.S.; CAMARGO, L.A.; TEIXEIRA, D.D.B.; BARRÓN, V.; TORRENT, J. Magnetic susceptibility and diffuse reflectance spectroscopy to characterize the spatial variability of soil properties in a Brazilian Haplustalf. Geoderma, v.219-220, p.63-71, 2014. DOI: 10.1016/j. geoderma.2013.12.007.

MATHÉ, V.; LÉVÊQUE, F.; MATHÉ, P.E.; CHEVALLIER, C.; PONS, Y. Soil anomaly mapping using a caesium magnetometer: limits in the low magnetic amplitude case. Journal of Applied Geophysics, v.58, p.202-217, 2006. DOI: 10.1016/j. jappgeo.2005.06.004.

MCBRATNEY, A.B.; MINASNY, B.; CATTLE, S.R.; VERVOORT, R.W. From pedotransfer functions to soil inference systems. Geoderma, v.109, p.41-73, 2002. DOI: 10.1016/ S0016-7061(02)00139-8.

NOVAES FILHO, J.P.; COUTO, E.G.; OLIVEIRA, V.A. de; JOHNSON, M.S.; LEHMANN, J.; RIHA, S.S. Variabilidade espacial de atributos físicos de solo usada na identificação de classes pedológicas de microbacias na Amazônia meridional. Revista Brasileira de Ciência do Solo, v.31, p.91-100, 2007. DOI: 10.1590/S0100-06832007000100010.

ROBERTSON, G.P. GS+: geostatistics for the environmental sciences - GS+ user's guide. Plainwell: Gamma Design Software, 1998. 152p.

ROLIM NETO, F.C.; SCHAEFER, C.E.G.R.; COSTA, L.M.; CORREAA, M.M.; FERNANDES FILHO, E.I.; IBRAIMO, M.M. Adsorção de fósforo, superfície específica e atributos mineralógicos em solos desenvolvidos de rochas vulcânicas do Alto Paranaíba (MG). Revista Brasileira de Ciência do Solo, v.28, p.953-964, 2004. DOI: 10.1590/S0100-06832004000600003.

SANTOS, H.G. dos; JACOMINE, P.K.T.; ANJOS, L.H.C. dos; OLIVEIRA, V.A. de; OLIVEIRA, J.B. de; COELHO, M.R.; LUMBRERAS, J.F.; CUNHA, T.J.F. (Ed.). Sistema brasileiro de classificação de solos. 2.ed. Rio de janeiro: Embrapa Solos, 2006. $306 \mathrm{p}$.

SILVA, A.F. da; HALMEMAN, R.J.; ZIMBACK, C.R.L. Dependência espacial de atributos diagnósticos para delimitação de classes de solos. Geociências, v.32, p.93-100, 2013. 
SIQUEIRA, D.S.; MARQUES JUNIOR, J.; MATIAS, S.S.R.; BARRÓN, V.; TORRENT, J.; BAFFA, O.; OLIVEIRA, L.C. de. Correlation of properties of Brazilian Haplustalfs with magnetic susceptibility measurements. Soil Use and Management, v.26, p.425-431, 2010. DOI: 10.1111/j.1475-2 743.2010.00294.x.

TORRENT, J.; BARRÓN, V. Diffuse reflectance spectroscopy. In: ULERY, A.L.; DREES, L.R. (Ed.). Methods of soil analysis. Part 5. Mineralogical methods. Madison: Soil Science Society of America, 2008. p.367-387. (SSSA book series, 5).

VALLADARES, G.S.; PEREIRA, M.G.; ANJOS, L.H.C. dos. Adsorção de fósforo em solos de argila de atividade baixa. Bragantia, v.62, p.111-118, 2003. DOI: 10.1590/ S0006-87052003000100014.
VEROSUB, K.L.; ROBERTS, A.P. Environmental magnetism: past, present and future. Journal of Geophysical Research, v.100, p.2175-2192, 1995. DOI: 10.1029/94JB02713.

VIEIRA, S.R.; HATFIELD, J.L.; NIELSEN, D.R.; BIGGAR, J.W. Geostatistical theory and application to variability of some agronomical properties. Hilgardia, v.51, p.1-75, 1983. DOI: 10.3733/hilg.v51n03p075.

WARRICK, A.W.; NIELSEN, D.R. Spatial variability of soil physical properties in the field. In: HILLEL, D. (Ed.). Applications of soil physics. New York: Academic, 1980. p.319-344. DOI: 10.1016/B978-0-12-348580-9.50018-3.

WYSZECKI, G.; STILES, W.S. Color science: concepts and methods, quantitative data and formulae. $2^{\text {nd }}$ ed. New York: J. Wiley, 1982. 976p.

Recebido em 24 de agosto de 2014 e aprovado em 28 de janeiro de 2015 